\title{
Sunitinib paves the way for targeted therapies in neuroendocrine tumors
}

\author{
Eric Raymond • Sandrine Faivre • Pascal Hammel • \\ Philippe Ruszniewski
}

Received: 18 October 2009 /Accepted: 22 October 2009 /Published online: 13 November 2009

(C) Springer-Verlag 2009

\begin{abstract}
Sunitinib demonstrating efficacy in pancreatic islet cell carcinomas will pave the way for further trials in other neuroendocrine tumor types such as carcinoid, poorly differentiated neuroendocrine disease, and several other endocrine tumors that are dependent on VEGF/VEGFR for angiogenesis. In addition, other drugs with distinct mechanisms of action, such as mTOR inhibitors, currently investigated in phase III trials, may also supply novel options in those diseases to control tumor growth and metastasis.
\end{abstract}

Keywords Sunitinib · Pancreatic carcinoma, VEGF.

VEGFR, targeted therapy $\cdot$ Angiogenesis

Phase I trials aiming characterizing drug safety offer the opportunity to identify hints of activity, pushing forward the development of novel anticancer agents. This may also give a chance to treat patients with hopeless "unmet needs" tumors. As a result, while tumor response in phase I trials remains a secondary endpoint, case reports of objective responses are now regarded as crucial signals of activity, stimulating and guiding further phase II/III studies toward potentially novel niche indications.

Sunitinib was one such anticancer agent. During the course of the phase I trial with sunitinib, we witnessed

\footnotetext{
E. Raymond $\cdot$ S. Faivre $\cdot$ P. Hammel $\cdot$ P. Ruszniewski

Department of Medical Oncology and Gastroenterology,

Beaujon University Hospital,

Clichy, France

E. Raymond $(\bowtie)$

Service Inter-Hospitalier de Cancérologie Beaujon-Bichat,

Hôpital Beaujon AP-HP,

100, boulevard du général Leclerc,

92110 Clichy, France

e-mail: eric.raymond@bjn.aphp.fr
}

strong antitumor activity as reflected by the unusually high number of objective responses in several tumor types [1]. Sunitinib works by blocking multiple molecular targets implicated in the growth, proliferation and metastatic spreading [2]. Two important targets, vascular endothelial growth factor receptor (VEGFR) and platelet-derived growth factor receptor (PDGFR), are expressed by many types of solid tumors and are thought to play a crucial role in angiogenesis [3]. Sunitinib also inhibits other targets important to tumor growth, including KIT, FLT3 and RET. Tumors that first entered in phase I trials and responded to sunitinib were those for which above kinases were shown to play a major role, highly angiogenic by nature, and mostly resistant to classical cytotoxic agents. First responses were observed in renal cell carcinoma and imatinib-resistant gastrointestinal stromal tumors, leading to phase II/III trials that subsequently demonstrated the efficacy of sunitinib in those two indications $[4,5]$.

Neuroendocrine tumors stand as poorly sensitive to chemotherapy but are highly angiogenic and dependent of VEGF/VEGFR activation [6]. In the RIP1-Tag2 transgenic mouse model of pancreatic islet cell carcinoma, sunitinib reduced tumor burden and increased animal survival by inhibiting the proliferation of VEGFR-dependent endothelial cell and by reducing the PDGFR-dependent pericyte coverage [7]. Success stories may sometimes be triggered by nothing more than a case report from a single patient. Among the 3 patients with neuroendocrine tumors entered in phase I trials primarily referred for tumor progression after several lines of chemotherapy, one exhibited an impressive partial response and two others experienced sustained tumor stabilizations [1]. Based on those data, a multicentre phase II trial was launched with sunitinib $(50 \mathrm{mg} /$ day $4 / 2)$ in patients with neuroendocrine tumors. In this trial, it was sticking that among 66 patients with 
advanced pancreatic islet cell carcinoma the objective response rate was $16.7 \%$ with $56.1 \%$ of patients experiencing tumor stabilization for more than 6 months, leading to a median time-to-tumor progression of 7.7 months [8].

This set the basis for the launching of a large international double blind phase III trial comparing $37.5 \mathrm{mg}$ sunitinib (continuous dosing) to placebo in patients with welldifferentiated pancreatic neuroendocrine tumors progressing within 6 months prior to the study entry. Preliminary results from this trial showed a median progression-free survival of 11.1 months in patients treated with sunitinib, compared with 5.5 months in patients treated with placebo (Hazard ratio $0.397, p<0.001$ ). These preliminary data were presented at the 11th World Congress on Gastrointestinal Cancer in Barcelona, Spain [9] and the ECCO/ESMO2009 in Berlin [10]. Adverse events were similar to those observed in other sunitinib studies and mainly consisted of neutropenia, hypertension, diarrhea, and hand-foot syndrome [11]. Results from this study suggest that sunitinib has substantial antitumor activity with a good safety profile in patients with pancreatic islet cell tumors.

These data underline several important points:

- The identification of novel indications requires paying a close attention to the primary mechanism of action of novel targeted therapy and to main tumor types that are dependent of this mechanism for survival. Tumor angiogenesis primed by hypoxia and/or VHL appears often dependent on VEGF/VEGFR activation and as a result appears exquisitely sensitive to VEGF and VEGFR inhibitors.

- Phase I trials may evidence drug activity providing that sufficient knowledge of the primary mechanisms of action has been identified. Enriching phase I trial by expanding the number of patients with target-dependent tumor types is a powerful tool in drug development, demonstrating proof of mechanisms and providing confidence for the launch of phase II/III studies.

- Targeted therapies may often identify novel niche indications corresponding to unmet medical needs and as such drive medical progress. This was the case for sunitinib that yielded novel therapeutic options in several tumor types including pancreatic neuroendocrine tumors for which a few years ago few treatments were available.

It is likely that sunitinib demonstrating efficacy in pancreatic islet cell carcinomas will pave the way of further trials in other neuroendocrine tumor types such as carcinoids, poorly differentiated neuroendocrine diseases, and several other endocrine tumors that are dependent on VEGF/VEGFR for angiogenesis. In addition, other drugs with distinct mechanisms of action, such as mTOR inhibitors, currently investigated in phase III trials may offer multiple medical options to control tumor growth and metastasis [12]. It is worth saying that, while medical choices were limited in past decades, current times appear quite exciting and novel therapies very promising for patients suffering neuroendocrine tumors.

\section{References}

1. Faivre S, Delbaldo C, Vera K, Robert C, Lozahic S, Lassau N et al (2006) Safety, pharmacokinetic, and antitumor activity of SU11248, a novel oral multitarget tyrosine kinase inhibitor, in patients with cancer. J Clin Oncol 24:25-35

2. Faivre S, Djelloul S, Raymond E (2006) New paradigms in anticancer therapy: targeting multiple signalling pathways with kinase inhibitors. Semin Oncol 33:407-420

3. Faivre S, Demetri G, Sargent W, Raymond E (2007) Molecular basis for sunitinib efficacy and future clinical development. Nat Rev Drug Discov 6(9):734-745

4. Motzer RJ, Hutson TE, Tomczak P, Michaelson MD, Bukowski RM, Rixe $O$ et al (2007) Sunitinib versus interferon $\alpha$ in metastatic renal-cell carcinoma. N Eng. J Med 356:115-124

5. Demetri GD, van Oosterom AT, Garrett CR, Blackstein ME, Shah MH, Verweij J et al (2006) Efficacy and safety of sunitinib in patients with advanced gastrointestinal stromal tumour after failure of imatinib: a randomized controlled trial. Lancet 368:1329-1338

6. Couvelard A, O'Toole D, Turley H, Leek R, Sauvanet A, Degott C et al (2005) Microvascular density and hypoxia-inducible factor pathway in pancreatic endocrine tumours: negative correlation of microvascular density and VEGF expression with tumour progression. Br J Cancer 92(1):94-101

7. Pietras K, Hanahan D (2005) A multitargeted, metronomic, and maximum-tolerated dose "chemo-switch" regimen is antiangiogenic, producing objective responses and survival benefit in a mouse model of cancer. J Clin Oncol 23:939-952

8. Kulke MH, Lenz HJ, Meropol NJ, Posey J, Ryan DP, Picus J et al (2008) Activity of sunitinib in patients with advanced neuroendocrine tumors. J Clin Oncol 26(20):3403-3410

9. Raymond E, Raoul JL, Niccoli P, Bang Y-J, Borbath Y, LombardBohas C et al (2009) Phase III, randomized, double-blind trial of sunitinib vs placebo in patients with progressive, welldifferentiated, malignant pancreatic islet cell tumors. Ann Oncol 20(10): abstract O-008

10. Raoul JL, Niccoli P, Bang Y-J, Borbath I, Lombard-Bohas C, Metrakos P et al (2009) Sunitinib (SU) vs placebo for treatment of progressive, well-differentiated pancreatic islet cell tumours: results of a phase III, randomised, double-blind trial. Eur J Cancer Suppl 7(2):361

11. Theou-Anton N, Faivre S, Dreyer C, Raymond E (2009) Benefit-risk assessment of sunitinib in gastrointestinal stromal tumours and renal cancer. Drug Saf 32(9):717-734

12. Yao JC, Phan AT, Chang DZ, Wolff RA, Hess K, Gupta S et al (2008) Efficacy of RAD001 (everolimus) and octreotide LAR in advanced low- to intermediate-grade neuroendocrine tumors: results of a phase II study. J Clin Oncol 26(26): $4311-4318$ 